\title{
Jürgen Habermas Wie ist Legitimität durch Legalität möglich?
}

Ich will die These verteidigen, daß die Autonomisierung des Rechtssystems keine vollständige Enckoppelung des Rechts von Moral einerseits, Politik andererseits bedeuteo kann. Auch das positiv gewordene Recht kappi nicht seine internen Beziehungen zu Moral und Politik. In einem ersten Teil werde ich grob skizzieren, wie sich das moderne Recht mit Hilfe des Vernunftrechts aus dem traditionellen Komplex von Moral, Recht und Politik ausdifferenziert hat. Im mirtleren Teil wird uns die Frage beschäfrigen, wie aus dem Zusammenbruch des Vernunftrechts eine Idee des Rechtsstaates hervorgeht, die einer Gesellschaft von hoher Komplexität und beschleunigtem Wandel nicht nur ohnmächtig gegenüberstehen muß. Im abschließenden Teil will ich aus einer internen Perspektive untersuchen, wie sich heute Recht und Moral gleichzeitig ergänzen und verschränken.

\section{Vernunft und Positivität: Zur Verschränkung von Recht, Politik und Moral}

(s) Wenn wir uns klar machen wollen, warum die Ausdifferenzierung des Reches dessen interne Verschränkung mit Politik und Moral keineswegs vollständig auflöst, empfiehlt sich ein Rückblick auf die Entstehung des positiven Rechts. Dieser Vorgang hat sich in Europa vom ausgehenden Mittelalter bis zu den großen Kodifikationen des r8. Jahrhunderts erstreckt. Auch in den Ländern des CommonLaw wird das Gewohnheitsrecht unter dem Einfluß akademisch gebildeter Juristen durch römisches Reche überformt; dabei wird es sukzessiv an Verkehrsbedingungen einer entstehenden kapitalistischen Wirtschaft und an die bürokraxisierte Hertschast des sich ausbildenden Territorialstaats angepaßt. Diesen verschlungenen, variantenreichen, schwer überschaubaren Prozeß will ich hier lediglich auf eine Pointe bringen, die in unserem rechtsphilosophischen Zusammenhang interessiert. Was dic Positivierung des Rechts philosophisch bedeutet, läßı sich vor dem Hintergrund der dreigliedrigen Struktur des zerfallenden mittelalterlichen Rechtssystems besser erklären.

Aus einer gewissen Distanz erkennt man in unseren heimischen Traditionen Entsprechungen zu jenen drei Elementen, die nach den Einsichcen der vergleicbenden Rechtssoziologie für die Rechtskultur der Alten Reiche typisch gewesen sind.' Das Rechtssystem wird jeweils überwölbt durch ein von theologischen und juristischen Fachleuten exegetisch verwaltetes sakrales Recht; scin Kernstück bildet das bürokracische, vom König oder Kaiser, der zugleich oberster Gerichtsherr ist, in Übereinstimmung mit den geheiligten Rechtstraditionen gesetzte Recht. Beide 
Rechustypen überformen das in der Regel ungeschriebene Gewohnheirsrecht, das in letzter Instanz auf scammesrechtliche Überlieferungen zurückgeht. Im europäischen Mjtrelalter lagen die Dinge insofern etwas anders, als das kanonische Reche der katholischen Kirche ohne Unterbrechung das hohe rechrstechnische und begriffliche Niveau des klassiscben römischen Rechts fortsetzte, während das Herrscherrecht der kaiserlichen Erlasse und Kapitularien auch schon vor der Wiederentdekkung des Corpus Juscinianum wenigstens an die Idee des römischen Imperiums anknüpfre. Selbst das Gewohnheitsrecht verdankte sich der gemischten römischgermanischen Rechtskultur der weströmischen Provinzen und prurde seit dem 12. Jahrhunderr schriftlich tradiert. Aber in den wesentlichen Zügen wiederholt sich die aus allen Hochkulturen bekannre Struktur - die Verzweigung in sakrales uod profanes Recht, wobei das sakrale Recht im Horizont einer der großen Weltreligionen der Ordnung des Kosmos oder dem Heilsgeschehen integriert ist. Dieses görtliche oder aarürliche Recht steht dem politischen Herrscher nicht zur Disposition. Es gibr vielmehr den legitimierenden Rahmen vor, innerhalb dessen der Herrscher über die Funktionen der Rechtsprechung und der bürokratischen Rechtsezzung seine profane Herrschaft ausübt. In diesem Zusammenhang spricht $M$. Weber vom "Doppeleeich der traditionalen Herrschaft $\alpha_{.}{ }^{2}$

Auch im Mitcelatter bleibr dieser traditionale Charakter des Rechts erhalten. Alles Recht entlehnt seinen Geltungsmodus der göttlichen Herkunft des christlich verstandenen Nanurrechts. Neues Rechr kann nur im Namen der Reformation oder Wiederherstellung des guter alten Rechts geschaffen werden. Die Bindung ans traditionale Rechtsverständnis enthält schon eine interessante Spannung, die zwischen den beiden Elementen des Herrscherrechts besteht. Als oberster Gerichtsherr untersteht der Herrscber dem sakraien Recht. Nur so kann sich dessen Legitimitär aú die weltliche Macht übertragen. Aus der pietärvolien Wahrung der unantastbaren Rechtsordnung erwächst eine Legitimitärsprämie für die Ausübung der politischen Herrschaft überhaupt. Zugleich macht aber der Herrscher an der Spizze einer nach Ämtern organisierten Verwaltung auch vom Recht a's einem Medium Gebrauch, das seinen Befehlen beispielsweise in der Form von Edikren und Erlassen kollektive Verbindlichkeit verleiht. Auf dieser Seice kann das Recht als Mittel der bürokraxischen Herrschafrsausübung allerdings nur so lange Ordnungsfunkrionen erfullen, wie es auf der anderen Seite in Gestalt geheiligzer Rechtstraditionen zugleich den nichrinstrumentellen, unverfügbaren Charakter behält, den der Herrscher in der Rechtsprechung respektieren muß. Zwischen diesen beiden Momenten - der Unverfügbarkeir des in der gerichtlichen Konfliktregelung vorausgesetzren Rechts, der Instrumentalitär des für die Herrschaftsordnung in Dienst genommenen Rechts - besteht eine unaufgelöste Spannung. Sie bleibt unauffällig, solange die sakrale Grundiage des Rechts nicht angefochten wird und der Sockel des traditionsfesten Gewohnheitsrechts in der Alltagspraxis fest verankert ist. ${ }^{3}$

(2) Wenn man nun davon ausgebt, daß in modernen Gesellschaften genau diese beiden Bedingungen immer weniger erfüllt werden können, kann man sich die Positivierung des Rechts als eine Reaktion auf solche Veränderungen erklären.t In dem Maße, wie die religiösen Welcbilder privarisierten Glaubensmächren weichen und die gewohnheitsrechtlichen Überlieferungen auf dem Wege über den usus modernus vom gelehrten Recht absorbiert werden, muß die dreigliedrige Struktur

2 Vgl. dazu W. Scbluchter, Die Entwicklung des okuzidentalen Rauonalismus (Tübingen 1980).

3 Vgl. H.Schlosser, Grundzüge der neveren Pryvatrechesgeschichee (Heidelberg r $9^{8}$ z).

4 Diesen internen Aspekt vemachlässıg die funktionalistische Deutung des Positivierungsschubs: vgl

N. Luhmann, Rechessoziologie (Opladen r 983 ). 
des Rechtssystems zerbrechen. Das Recht schrumpft auf eine Dimension zusammen und beserzt nur noch den Platz, den bis dahin das bürokratische Herrscherrecht eingenommen hatre. Die politische Gewait des Herrschers emanzipiert sich von der Bindung ans sakrale Recht und wird souverän. Ihr fällt die Aufgabe zu, die Lücke, die das theologisch verwaltete Naturrecht hinterlassen hat, aus eigener Krafs durch politische Gesetzgebung zu füllen. Fortan fließt alles Reche aus dem souveränen Wullen des politischen Gesetzgebers. Gesetzgebung, Vollzug und Anwendung der Gesetze werden zu drei Momenten innerhalb eines einzigen, politisch gesteuerten Kreisprozesses; sie bleiben es auch dann, wenn sie sich institutionell nacb Staatsgewalien differenzieren.

Dadurch verändert sich das Verhältnis jener beiden Momente der Unverfügbarkeit und der Instrumentalizät des Rechts. Bei hinreichender Differenzierung der Rollen, darin liegt ja die Bedeutung der Gewaltenteilung, werden die Geseczesprogramme zwar der Rechtsprechung vorgegeben. Aber kann vom beliebig änderbaren politischen Recht noch eine ähnlich verpflichtende Autorität ausgehen wie zuvor vom unverfügbaren sakralen Recht? Bewahrt das posicive Reche überhaupt noch einen verpflichtenden Charakter, wenn es seinen Gelcungsmodus nicht mehr, wie das bürokratische Herrscherrecht im traditionalen Rechessystem, einem vorgängigen und übergeordnecen Recht entlehnen kann? Auf diese Fragen hat der Rechrspositivismus immer wieder affirmative Antworten gegeben.' In der einen Variante wird das Reche überhaupt seines normativen Charakters beraubt und nur noch instrumentell definiert: Es gilt als der Befehl eines Souveräns (Austin). Dabei verschwindet das Moment der Unverfügbarkeit als mecaphysisches Relikt. Die andere Variante des Rechtspositivismus hält fest an der Prämisse, daß das Recht seine Kernfunktion der gerichtlichen Konfliktregelung nur solange erfüllen kann, wie den angewendeten Gesetzen ein Moment von Unverfügbarkeit erhalten bleibt. Aber dieses Moment darf nun nur noch an der Form des positiven Rechts, nicht meht an narurrechtlichen Inhalten haften (Kelsen). Aus dieser Sicht bleibt das von Politik und Moral getrennte Rechussystem, mit der Rechrsprechung als seinem institutionellen Kern, als der einzige Ort zurück, wo das Recht aus eigener Kraft seine Form und damit seine Autonomie wahren kann. Beide Male ergibt sich die Konsequenz, daß die durchs sakrale Recht gegebene metasoziale Gazancie der Rechesgeltung ersatzlos entfallen kann.

Die historischen Ursprünge des modernen wie des waditionalen Rechts sprechen gegen diese These. Recht überhaupt geht nämlich der Entscehung politischer, d.h. staatlich organisierter Herrschaft voraus, während staatlich sanktioniertes Recht und rech lich organisierte Staatsgewalt in der Form politischer Herrscbaft gleichzeirig entstetien. ${ }^{6}$ Es scheint so zu sein, daß die archaische Rechtsentwicklung das Auftreten einer politischen Herrschaftsgewalt, in der staatliche Macht und staatliches Recht sicb wectuselseitig konstituieren, erst ermöglicht hat. Bei dieser Konstellacion läßt sich schwer vorstellen, daß jemals das Recht entweder durch die Politik vollständig aufgesaugt oder von der Politik ganz abgespalten werden könnte. Zudem läßt sich zeigen, daß bestimmte Strukturen des moralischen Bewußtseins beim Zustandekommen der Symbiose von Recht und staaclicher Macht eine wichtige Rolle gespielt baben. Eine ähnliche Rolle spielt das moralische Bewußtsein beim Übergang vom traditionalen Recht zum profanen, durchs staatliche Gewaltmonopol abgesicherten und dem politischen Geserzgeber zur Disposition gestellten positiven Recht. Jenes Moment Unverfügbarkeit, das auch noch im modernen Recht

s N. Hörster, Recht und Moral (Götsungen 1972).

6 Zum Folgenden rgi. U. Wescl, Frühformen des Rechus (Frankfurt 1984). 
ein unverzichtbares Gegengewicht bilder zur polirischen Instrumentalisierung des Rechrsmediums, verdankt sich der Verschränkung von Politik und Recht mit Moral.

(3) Diese Konstellation stellt sich zum ersten Mal her mir der Symbiose von Rechz und staatlicher Macht. In neolithischen Stammesgeselischaften' sind typischerweise drei Mechanismen für die Regelung innerer Konflikte in Krafr: Praktiken der Selbsthilfe (Fehde und Blutrache), die rituelle Anrufung magischer Mächte (Orakel, Zweikampf) und die schiedsrichterliche Vermittlung als friedliches Äquivaleot für Gewalt und Zauberei. Solchen Vermitrlern feht noch die Kompetenz, den Streit der Parteien bindend oder autoritativ zu entscheiden und das Ureil gegen verwandtschaftliche Loyalizäten durchzusetzen. Neben dem Merkmal der Erzwingbarkeit fehlen auch Gerichsshöfe und Gerichtsverfahren. Zudem bleibt das Recht mit Sitte und religiösen Vorstellungen noch so eng verschwistert, daß genuin rechtliche von anderen Phänomenen schwer abzugrenzen sind. Das allen Formen der Konfliktregelung zugrundeliegende Gerechtigkeitskonzept ist in die mythische Deutung der Welt verwoben. Rache, Vergeltung, Entschädigung dienen der Wrederherstellung einer gestörten Ordnung. Diese aus Symmetrien und Gegenseitigkeiten konstruierte Ordnung erstreckt sich gleichermaßen auf einzelne Personen und Verwandischaftsgruppen wie auf Natur und Gesellschaft im ganzen. Die Schwere des Verbrechens bemißt sich an den Konsequenzen der Tat, nichı an den Intentionen des Täters. Eine Sankvion hat den Sinn einer Kompensation des entstandenen Schadens, nicht den der Bestrafung eines Täters, der sich einer Normverletzung schuldig gemacht hat. Diese konkreciscischen Gerechrigkeitsvorstellungen erlauben noch nichr die Trennung zwischen Rechts- und Tatsacheofragen. Im archaischen Rechusgang fließen normacive Urteile, kJuge Interessenabwägungen und Tatsachenbehauptungen ineinander. Es fehlen Begriffe wie Zurechnungsfähigkeit und Schuld; Vorsatz und Fahrlässigkeit werden nicht unterschieden. Was zählt, ist der objekriv verursachte Schaden. Es gibc keine Trennung von Privatrecht und Strafrecht; alle Rechtsverletzungen sind gewissermaßen Delikte, die Schadensersatz verlangen. Solche Unterscheidungen werden erst möglich, wenn ein ganz neues Konzept aufritr und die Welt der moralischen Vorstellungen revolutioniert. Ich meine den Begriff der sicuationsunabbängigen, den streitenden Parteien wie auch dem unparteilichen Schiedsrichter übergeordneten, also vorgängig und generell als verbindlich anerkannten Rechtsnorm. Um diesen Kern kristallisiert sich das, was L. Kohlberg ein konventionelles moralisches Bewußusein nennc. Ohne ein solches Normkonzept kann der Schiedsrichter die streitendeo Parteien nur dazu überreden, Kompromisse zu schließen. Dabei mag er das persönliche Ansehen, das er seinem Stacus, seinem Reichrum oder seinem. Alter verdankt, als Einfluß geltend machen. Aber politische Macht fchlt ihm; an die unpersönlich verpflichtende Autorität eines Gesetzes und die moralische Einsicht der Beceiligten kann er noch nicht appellieren. ${ }^{8}$

Ich schlage nun folgendes Gedankenexperiment vor: Nehmen wir einmal an, daß sich, bevor so enwas wie eine staatliche Autorität entsteht, konventionelle Rechtsund Moralvorstellungen bilden. Dann könnte sich beispielsweise ein streitschlichtender Häupdling schon auf den verpflichrenden Charakrer anerkannter Rechtsnormen stützen; aber der moralischen Verbindlichkeit seines Urteils könnte er noch nicht den fakrisch zwingenden Charakter eines staadichen Sanktionspotentials hinzufügen. Dennoch müßze sich die Rolle des Häuptlings, dessen Fübrungsrolle

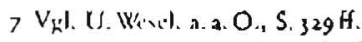

8 L. Pospisil, Anthropologie des Reclies. Reche und Gescllschaft in archaischen und modernen Kulturen (Munchen 1982). 
bis dahin allein auf faktischem Einfluß und Prestige beruhre, folgenreich ändern.

Drei Sequenzen sind wichtig in diesem Szenario. Ein solcher Häuptling härte als Wahrer intersubjektiv anerkannter Normen erstens teil an der Aura des von ihm verwalteten Rechts. Die normative Autorität des Reches könnte sich von der Kompetenz des Richters auf die damic in Personalunion verbundene Befehlsmacht des Häupelings im ganzen übertragen. Unmerklich verwandelce sich dann die fakrische Macht des Einflußreichen in die normaciv autorisierte Macht eines Befehlshabers, der nun kollektiv bindende Entscheidungen treffen kann. Daraufhin müßte sich zweitens die Qualität der richterlichen Entscheidung selbst ändern. Hinter den moralisch verpflichtenden Rechtsnormen stünde nicht mehr nur der Konformitätsdruck des Stammes oder der faktische Einfluß eines Prominenten, sondern die Sanktionsdrohung eines legitimen Herrschers. So entstünde der ambivalente, Anerkennung und $Z$ wang fusionierende Geltungsmodus scaatlichen Rechts. Mit diesem würde aber der politische Herrscher dorittens ein Medium gewinnen, mit dessen Hilfe er eine Ämterorganisation schaffen und seine Herrschaft bürokratisch ausüben kann. Als Organisationsmitcel erhält dadurch das Recht neben dem Aspekt der Unverfügbarkeit objektiven Rechts auch einen instrumentellen Aspekt.

Obwohl diese Überlegungen auch einen empirischen Gehalt haben', geht es mir in erster Linie um die Klärung konzeptueller Beziehungen. Erst in komplexer werdenden Weltbildern bildet sich ein moralisches Bewußrsein konventioneller Stufe aus; erst ein Bewußtsein von traditional verankerten und moralisch verpflichtenden Normen ermöglicht die Umwandlung von faktischer Macht in normative; erst die Verfügung über legitime Macht erlaubt die politische Durchsetzung von Rechtsnormen; erst zwingendes Recht kann für die Organisation staatlicber Macht genutzt werden. Wenn man diese Verschränkung von religiös eingebetteter Moral, rechtlich legitimierter Herrschaft und rechtsförmig organisierter staadicher Verwalung im einzelnen analysiert, wird die Unhaltbartseit der beiden genannten positivistischen Recheskonzepte klar.

(4) Die Reduktion von Rechrsnormen auf Befehle des politischen Gesetzgebers bedeutet, daß sich Recht in Politik gleichsam auflöst. Damit zersetzt sich aber der Begriff des Politischen selbst. Unter dieser Prämisse kann jedenfalls politiscbe Herrschaft niche mehr als rechtlich legitimierze Macht verstanden werden; denn ein der Policik vollständig verfügbar gewordenes Recht verliert seine legitimierende Kraft. Sobald Legitimation als Eigenleistung der Politik vorgestellt wird, geben wir unsere Begriffe von Recht und Politik auf. Die gleiche Konsequenz ergibt sich für die andere Auffassung, daß das positive Recht seine Autonomie aus eigener Kraft, d.h. durch die dogmatischen Leistungen einer gesetzestreven, aber gegenüber Politik und Moral verselbständigten Justiz aufrechrerhalten könne. Sobald die Rechtsgeltung jeden über die Dezision des Gesetzgebers hinausgreifenden moralischen Bezug zu Aspekten der Gerechrigkeit einbüßc, wird die Idencität des Recbrs selber diffus. Dann fehlen nämlich die legitimierenden Gesichtspunkte, unter denen das Rechissystem auf die Bewahrung einer bestimmten Struktur festgelegr werden könnte.

Vorausgesetzt, daß moderne Gesellschaften nicht überhaupe auf Recht verzichten (auch nicht unter dem beibehaltenen Pseudonym von "Recht e eine funktional äquivalente, aber ganz andere Art von Praxis betreiben) können, schafft die Positivierung des Rechts schon aus konzepruellen Gründen ein Folgeproblem. Für das entzauberte sakrale Recht - und für ein entleertes, substanzlos gewordenes Ge-

9 K.Eder, Die Entstehung staatich organisierter Gesellschaften (Frantefurc 1976); J. Haberuas, Zur Rekonseruktion des Historischen Marerialismus (Frankfur 1976). 
wohnheirsrecht - muß ein Äquivalent gefunden werden, welches dem positjven Recht ein Moment Unverfügbarkeit erhalcen kann. Ein solches Äquivalent ist zunächst in Gestalt des Vermunftreches entwickelt worden, das ja nichr nur rechisphilosopbische, sondern für die großen Kodifikationen und die richterliche Praxis der Rechtsfortbildung eine unmittelbar rechtsdogmatische Bedeutung gehabt hat. ${ }^{10}$ Ich möchte in unserem Zusammenhang die Aufmerksamkeit auf zwei Punkte lenken: a) Im Vernunftrecht artikuliert sich eine neue, posttraditionale Stufe des moralischen Bewußuseins, die das moderne Recht von Prinzipien abhängig mache und auf Verfahrensrationalität umstellt. b) Je nachdem, ob die Positivierung des Rechts als solche oder ob der daraus entstandene Begründungsbedarf als erklärungsbedürfuges Phänomen in den Vordergrund rückte, sind die Sozialvertragstheorien in entgegengeserzten Richtungen enrwickelt wordeo. So oder so haben sie aber zwischen den Momenten der Unverfügbarkeit und der Instrumeacalität des Recbrs keine plausible Beziehung herstellen können.

ad a). Das Vernunftreche reagierc auf den Zerfall des religiös und metaphysisch begründeren Narurrechts und auf die Entmoralisierung einer zunehmend naturaliscisch gedeuteren, auf Selbstbebauptungsinteressen umgestellten Politik. Sobald sich der gewaltmonopolisierende Staat in der Rolle des souveränen Gesetzgebers einen exklusiven Zugriff aufs Recht verschafft, droht dem zum Organisationsmittel herabgesetzten Recht jeder Bezug zur Gerechrigkeit, und damit der genuine Rechtscharakter, verloren zu gehen. Mit der Positivität des vom staaclichen Souverän abhängig gewordenen Rechts verschwindet die Begründungsproblematik nicht, sie verschiebt sich nur auf das scbmaler gewordene Fundament einer nachmetaphysischen, von Weltbildern entkoppelten Profanethik.

Die Grundfigur des bürgerlichen Privatreches ist der Vertrag. Die Vertragsautonomie ermächtigt private Rechrspersonen zur Erzeugung subjektiver Rechte. In der Idee des Gesellschaftsvertrages wird nun diese Denkfigur in einer interessanten Weise benutzt, um die in Formen des positiven Rechts ausgeübte Herrschaft - legale Herrschaft - moralisch zu rechrfertigen: Ein Vertrag, den jeder autonome Einzelne mit allen anderen autonomen Eiozelnen abschließt, kann nur etwas zum Inhalt haben, was alle im jeweils eigenen Interesse vernünfrigerweise wollen können. Auf diesem Wege können nur solche Regelungen zuscande kommen, die die ungezwungene Zuscimmung aller haben. Dieser Grundgedanke verrät, daß die Vernunft des modernen Naturrechts wesentlich praktische Vernunif ist - die Vernunft einer autonomen Moral. Diese verlangt, daß wir zwischen Normen, rechtferrigenden Prinzipien und Verfahren unterscheiden - Verfahren, nach denen wir prüfen, ob Normen im Lichte gültiger Prinzipien auf allgemeine Zuscimmung rechnen dürfen. Indem mir der Idee des Gesellschafrsvertrages ein solches Verfahren für die Begründung rechtlich verfaßter politischer Ordnungen in Anschlag gebracht wird, wird das positive Recht moralischen Grundsätzen unterworfen. Aus entwicklungsgeschichtlicher Perspektive lege das die Hypochese nahe, daß beim Übergang zur Modeme wiederum eine Veränderung des moralischen Bewußtseins Schritrmacberfunkcionen für die Rechtsentwickluog erfüllt bat.

ad b). Das Vernunftrecht ist in verschiedenen Versionen aufgetreten. Autoren wie Hobbes sind stärker vom Phänomen der beliebigen Änderbarkejh, Autoren wie Kant vom Begründungsdefizit des neuen positiv gewordenen Rechts fasziniert. Hobbes entwickelt seine Theorie bekannelich unter Prämissen, die sowohl dem positiven Recht wie der politischen Macht alle moralischen Konnotationen abstreifen; das vom Souverän geseczte Recht soll auch ohne ein vernünftiges Äquivalent für 
das entzauberte sakrale Recht auskommen können. Natürlich verwickelt sich Hobbes mit einer Theorie, die ibren Adressaten eben ein solches vernünfriges Äquivaleat anbietec, in einen performaciven Widerspruch. Der manifeste Gehalt seiner Theorie, die das moralfreie Funktionieren des vollscändig positivierten Rechts erklär, gerät in Widerspruch zur pragmatischen Rolle derselben Theorie, die ja ihren Lesern erklären will, warum sie als treie und gleiche Bürger gure Gründe haben könnten, sich für die Unterwerfung unter eine absolute Staatsgewalt zu entscheiden.

Kant macht später die von Hobbes implizit mitgeführten normativen Annahmen explizit und entrickelt seine Rechtslehre von Anbeginn im Rahmen der Moraltheorie. Das allgemeine Rechtsprinzip, das aller Gesetzgebung objekriv zugrundeliegt, ergibr sich aus dem Kategorischen Imperativ. Aus diesem obersten Prinzip der Gesetzgebung folgt wiederum das ursprüngliche subjektive Recht eines jeden, jeden anderen Rechrsgenossen dazu zu verpflichten, seine Freiheit zu respektieren, sofern sie nur mit der gleichen Freiheic aller nach allgemeinen Geserzen zusammenscimmt. Während für Hobbes das positive Recht letztlich ein Organisationsmittel der politischen Herrschaft ist, behält es für Kant einen wesentich moralischen Charakter. Aber auch in diesen ausgereiftesten Versionen tur sich das Vernunítrechr schwer mit der selbstgestelizen Aufgabe, die Legitimitätsbedingungen legaler Herrschaft vernünfrig zu erklären. Hobbes opfert die Unverfügbarkeit des Rechts seiner Positivität auf, bei Kant gewinnt das aus praktischer Vernunft apriori abgeleitete narürliche oder moralische Reche so sehr die Oberhand, daß Reche in Moral aufzugehen droht: Reche wird zu einem defizienten Modus der Moral herabgestuft.

Kant baut das Moment Unverfügbarkeic in die moralischen Grundlagen des Rechts derart ein, daß das positive Recht dern Vernunftrecht subsumiert wird. In diesem vernunfrrechtlich präjudizierten Recht bleibr kein Raum für den instrumencellen Aspekt eines Rechts, dessen sich der policische Gesetzgeber für seine Gestaltungsaufgaben bedient. Nachdem der Baidachin des christlichen Naturrechts eingestürzt iš, sind die Säulen der naturalistisch ernüchterten Politik auf der eioen, des auf politische Dezision umgestellen Rechts auf der anderen Seite als Ruinen stehen geblieben. Kant rekonstruiert das zerfallene Gebäude durch einfache Substitution: Das autonom begründete Vernunfurecht soll den vakanten Platz des religiösmetaphysischen Naturrechts einnehmen. Damit ändert sich im Vergleich zum dreigliedrigen zraditionalen Recht $z w a r$ die vermiztelnde Funktion der Rechtsprechung, die die sakuale Legitimation auf den Herrscher und seine bürokratische Herrschaft übertragen hatte; sie tritt nun hinter den politischen Gesetzgeber zurück und verwaltet dessen Programme. Jetzt geraten aber die in sich differenzierten Staatsgewalten allesamt in den Schatten einer aus Vernunft gerechtfertigten res publica noumenon, die in der res publica phaenomenon ein möglichst gesreues Abbild finden soll. Die Positivierung des Rechis steht als die Realisierung vernunferechtlicher Grundsätze selbst noch uncer Imperativen der Vernunuft.

Wenn aber Politik und Recht in die subordinierte Stellung von Ausführungsorganen für die Gesetze der praktischen Vernunft geschoben werden, verliert die Politik ihre gesetzgeberische Kompetenz und das Recht seine Posicivicät. Deshalb muß Kant auf die metaphysischen Prämissen seiner Zwei-Reiche-Lehre zurückgreifen, um Legalität von Moralităt auf eine höchst widerspruchsvolle Weise voneinander zu unterscheiden."

i) Wersuing. Wohlgeordnete Freibeir (Berlin 1984), 16ff. 
(1) Das Vernunftrecht ist nicht nur aus philosophischen Gründen preisgegeben worden; die Verhältnisse, die es interprecieren sollte, sind ihm über den Kopf gewachsen. Bald wurde $k$ lar, daß sich die Dynamik einer über Märkte integrierten Gesellschaft in den normariven Begriffen des Rechts nicht mehr einfangen und im Rahmen eines apriorisch entworfenen Rechtssystems erst recht nicht scillstellen ließ. Jeder Versuch, die Grundlagen des privaten und des öffentlichen Rechts theoretisch ein für allemal aus obersten Prinzipien abzuleiten, mußte an der Komplexität von Gesellschaft und Geschichce scheitern. Die Vertragstheorien - und keineswegs nur dje idealistischen unter ihnen - waren zu abstrakt angelegt. Sie hatren sich über die sozialen Vorausseczungen ihres possessiven Individualismus keine Rechenschaft abgelegt. Sie harten sich nichr eingestanden, daß die grundlegenden privarrechtlichen Instirute Eigencum und Vertrag wie auch die subjekriv-öffentlichen Abwehrrechre gegen den bürokratischen Staat nur durch das Entgegenkommen einer fikriven Kleinwarenwirtschaft Gerechtigkeit verheißen konnten. Gleichzeitig waren die Vertragstheorien - und keineswegs nur die aprioristisch verfahrenden - zu konkretiscisch angelegt. Sie harren sich über die Mobilisierung der Lebensverhältnisse keine Rechenschaft abgelegt und den Anpassungsdruck unterschätzt, der vom kapitalistischen Wachstum, von gesellschafclicher Modernisierung überhaupt ausging.

In Deutschland ist der moralische Gehalt des Vermunftrechts von der Rechtstheorie aufgespadten und auf den parallelen Gleisen der Privatrechtsdogmatik und der Rechtsstaatsidee zunächst fortgeführt, im Verlaufe des 19. Jahrhunderts aber positivistisch ausgetrocknet worden. Aus der Sichc der Pandektenwissenschaft ging das Recht wesentlich in dem von Juristen verwalteten zivilrechtlichen Kodex auf. Hier, im Privacrechtssystem sclbst, und nicht von seiten eines demokracischen Gesetzgebers, sollten die moralischen Gehalte des Rechis gesichert werden. " F. C. von Savigny, der das gesamte Privatrecht als ein Gebäude von subjekriven Rechten konscruierte, war in Anlehnung an Kant der Auffassung, daß die Form des subjektiven Rechts an sich selbst morabsch sei. Aligemeine subjektive Rechte grenzen privatautonome Verfügungsbereiche aus und garantieren auf dem Wege subjektiver Berechtigungen individuelle Freiheit. Die Moralität des Rechts besteht darin, "daß dem individuellen Wullen ein Gebiet angewiesen wird, in welchem er unabhängig von jedem fremden Willen zu herrschen hat. ${ }^{13}$ Aus der fakrischen Rechtsentwicklung wurde aber bald klar, daß subjekrive Rechte etwas gegenüber dem objektiven Rechc Sekundäres sind und auch beineswegs die konzepruelle Grundlage für das Privatrechtssystem im ganzen bieten können. Der Begriff des subjektiven Reches ist daraufhin positivistisch ungedeutet und von allen normaciven Assoziationen gereinigt worden. Nach B. Windscheids Definition setzen subjektive Rechte nur noch die Befehle der objekciven Rechtsordnung in die Befehlsmacht einzelner Rechissubjekte um.

Eine parallele Entwicklung läßt sich für die Idee des Rechosstartes nachzeichuen, die Kant ohnehin nur unter hypothetischen Vorbehalteo eingeführt batte. Die deutschen Theoreriker des 19. Jahrhunders sind vor allem an der konstitutionellen Zähmung der monarchischen Verwaltungsmacht interessiert. Mohl und Welcler setzen im Vormärz noch darauf, daß sich allgemeine und abstrakte Gesetze als das

12 H. Coing, Das Verhälnis der positiucn Rechtswissenschah zur Ethik im rg. Jabrbuodere, in: J. Blübdorn/J. Ritter ( $\mathrm{Hg}_{\mathrm{g}}$ ). Recht und Echik (Frankturt 1970), a $1 \mathrm{H}$.

13 F.C. von Savigny, System des heucigen Römischen Reehis [ (1840), 33$\}$. 
geeignete Medium erweisen für eine gleichmäßige Förderung aller Staatsbürger »bei der möglichst allseirigen, vernunftgemäßen Ausbildung sämtlicher geisciger und körperlicher Kräfte. "' Nach der Reichsgründung entwickeln Gerber und Laband bereits die Lehre vom Geserz als dem Befehl einer souveränen, inhaltlich ungebundenen Geserzgebungsinstanz. Es ist dieser positivistische Geseczesbegriff, der schließlich von progressiven Staassrechtlern der Weimarer Zeit wie Heller für den parlamencarischen Gesetzgeber in Anspruch genommen wurde: „Gesetze heißen im Rechtsstaat nur, aber auch alle von der Volkslegislative geserzen Rechtsnormen. $\alpha^{\text {'s }}$

Ich erinnere an die gewiß nicht cypische deussche Encwicklung nur deshalb, weil sich hier die Erosion eines vernunftrechtich moralisierten Geserzesbegriffs aus der zweifachen Perspektive des Rechtsdogmatikers und Richters einerseits, des nach und nach pariamentarisierten Geseczgebers andererseits studieren läßt. In den angelsächsischen Ländern, wo die Idee des Rechtsstaates von Anbeginn im Einklang mit demokratischen Entwicklungen als s rule of law entfaltet worden ist, bot sich das faire Gerichtsverfahren - fair trial and due process - als einheitliches Interpretacionsmodell an, das auf Geserzgebung und Rechtsprechung zugleich angewendet worden ist. In Deutschland hat sich die positivistische Destrukuion des Vernunftrechts auf getrennten Wegen vollzogen. Gewiß, sowohl in der Privatrechrdogmatik wie in der Rechtsstaatstheorie wird Kants Konstruktion, nach der Politik und Reche den moralischen Imperativen des Vemunftrechis unterworfen waren, dementiert aber einmal aus der Sicht der Jusciz, das andere Mal aus der Sicht des politischen Gesetzgebers. Deshalb mußte sich für diejenigen, die nach dem Einsturz der vernunftrechtlichen Dachkonstruktion auch von der Alternative des schieren Rechespositivismus njcht überzeug waren, dasselbe Problem auf beiden Seiten in jeweils anderer form präsenticren.

Dem Problem kann man allgemein die folgende Fassung geben. Einerseits lassen sich die moralischen Grundlagen des positiven Rechts nicht in Gestajt eines übergeordneten Vernunftrechis erklären. Andererseits lassen sie sich auch nicht ersatzlos liquidieren, ohne dem Recht das ihm wesentlich innewohnende Moment von Unverfügbarkeit zu nehmen. Dann muß aber gezeigr werden, wie im Inneren des positiven Reches selbst der moralische Gesichtspunkt einer unparteilichen Urreils- und Willensbildung stabilisiert werden kann. Dieser Fordenung ist nicht schon dadurch Genüge getan, daß bestimmte moralische Prinzipien des Vernunftrechis als Inhalce des Verfassungsrechts positiviert werden. Denn um die Kontingenz der Inhalce eines beliebig änderbaren Rechts geht es gerade. Die ins positive Rechs eingebaute Moralität hat vielmehr die transzendierende Kraft eines sich selbst regulierenden Verfahrens, das seine eigene Vernünftigkeic kontrolliert.

(2) Unter dem Druck dieses Problems baben jene unter den Nacbfolgern Savignys, die sich mit der positivistischen Umdeutung subjektiver Rechte nicht zufrieden geben wollten, das wissenschaftliche Juristenrecht zur Legicimationsquelle ausgebaut. Savigny hatre in seiner Rechrsquellenlehre der Justiz und der Rechrsdogmatik noch die bescheidene und abgeleitete Funtrion zugewiesen, das aus Gewohnheic und Gesetzgebung stammende positive Recht sin wissenschaftlicher Weise zu Bewußtsein zu bringen und darzustellen ${ }^{16}$ Hingegen vertritr G. F. Puchea gegen Ende des Jahrhunderts die Auffassung, daß die Produktion von Recht nicht allein

${ }_{4} \mathrm{VgL}$ I. Maus, Entwickelung und Funkuonswandel des bürgerlichen Rechøscaats, in: M. Tohidipur ( $\mathrm{Hg}_{\mathrm{g}}$ ), Der bürgerlicbe Rechcsscaat I (Frankfurr 1978), 1 ; ff.

is H. Heller, Ges. Schrifien II (Leiden 1971), 226.

I6 F.C. von Savigny, Allgemeine Narur der Rechesquellen (1840), zIt. nach: W. Maihofer (Hg.), Begriff und Wesen des Reches (Darmstade 1973), 44. 
Sache des politischen Gesetzgebers sein dürfe, weil sonst der Staat nicht auf „Recht $"$ gegründet, $d . h$. Rechtsstaat sein könne. Vielmehr übernehme die Justiz, hinausgehend über die Anwendung gelrenden Rechrs, die produkcive Aufgabe einer von Prinzipien geleiceten konstrukciven Fortbildung und Ergänzung des geltenden Rechts." Dieses Richterrecht sollte seine unabhängige Autorität aus der wissenschaftlichen Mechode der Begründung, also aus den Argumenten einer wissenschaftlich verfahrenden Jurisprudenz beziehen. Bereies Puchta biezet den Ansatzpunkt für eine Theorie, die aus der Perspekrive der Rechrsprechung die legitimierenden Gründe der Legalizät auf die in den juriscischen Diskurs eingebaute Verfahrensrationalizät zurückführt.

Aus der Perspektive der Gesetzgebung legt sicb eine analoge Deurung nahe, auch wenn die parlamentarische Diskussion unmitrelbar auf Kompromißbildung und nicht, wie der juristische Diskurs, auf die wissenschaftlich disziplinierte Begründung von Ureilen angelegr ist. Auch auf dieser Seite stellt sich für diejenigen, die sich mit dem demokratischen Gesetzespositivismus nicht abfinden wollen, die Frage, aus welchen Gründen die mit parlamentarischen Mehrheiten zustandegekommenen Gesetze Legitimität beanspruchen dürfen. Schon Kant hatte im Anschluß an Rousseaus Begriff der Auronomie einen ersten Schritr getan, um aus dem Verfahren der demokrasischen Gesetzgebung selbst den moralischen Gesichrspunkt der Unparreilichkeir herauszuarbeiten. Zum Probierstein der Rechtmäßigkeir eines jeden öffentlichen Gesetzes erklär er bekanntlich das Kriterium, ob dieses naus dem vereinigten Willen eines ganzen Volkes habe entspringen können ${ }^{18}{ }^{18}$ Freilich hat Kant selbst dazu beigecragen, daß alsbald zorei ganz verschiedene Bedeutungen von „Allgemeinheit « des Gesetzes verwechselt wurden: Die semantische Allgemeinheit des abstrakt allgemeinen Gesetzes trat an die Stelle jener prozeduralen Allgerneinheir, die das demokratisch zustandegekommene Gesetz als Ausdruck des "vereinigten. Volkswillens\& auszeichner.

In Deurschland, wo die demokratietheoretische Diskussion obnehin erst wieder in den zoer Jahren auflebre, hat diese Verwechslung zwei mißliche Konsequenzen gehabt. Zum einen konnte man sich über die unständlichen Beweislasten einer prozeduraliscisch angelegten Demokratietbeoric täusehen, die erst noch abzutragen sind. Erstens müßte argumentationstheorecisch gezeigt werden, wie in der parlamentarischen Willensbildung des Geseczgebers politische Zielsetzungs- und moralische Begründungsdiskurse mit rechtlicher Normenkontrolle ineinandergreifen. Zweitens müßte klargemacht werden, worin sich ein argumentativ erzieltes Einverständnis von Verhandlungskompromissen uncerscheidet und wie sich der moralische Gesichtspunkt wiederum in den Fairneßbedingungen für Kompromisse zur Geltung bringt. Drittens und vor allem müßte aber rekonstruiert werden, wie die Unparteilichkeic der gesetzgeberischen Willensbildung durch rechtliche Verfahren institucionalisiert werden soll - angefangen von der Mehrheitsregel über die Regeln der parlamentarischen Geschäftsordnung bis zum Wahlrecht und der Meinungsbildung in der politischen Öffentlichkeit. Diese Analyse müßre sich von einem Modell leiten lassen, das die notwendigen Kommunikationsvoraussetzungen für diskursive Willensbildung und fairen Ausgleich von Interessen in ibrem Zusammenhang darstellt. Nur auf einer solchen Folie läßt sich der normative Sinn und die tarsächliche Praxis solcher Verfahren kritisch analysieren.'

Weicerhin hatte aber jene Verwechslung der prozeduralen Allgemeinheit mit der

17 G. F. Puchta, Vom Rechr $\left(188_{4} 1\right)$, zil nach Maihofer, a. a. O. S. $52 \mathrm{ff}$.

I8 I. Xank, Grundloguog der Metaphysik der Sitren.

19 U. Neumann, Jurisasche Argumentationstebre (Darmsede 1986), $70 \mathrm{ff}$. 
semantischen Allgemeinheit des parlamentarischen Geseczes die Konsequenz, daß man sich über die eigenständige Problematik der Rechtsanwendung täuschen konnte. Selbse wenn die moralisch gehaltwolle Verfabrensrationalicät der Geseczgebung institutionell hinreichend gesichert wäre, hätten die Gesetze, ob es sich nun ums regulatorische Recht des Sozialstaates handelt oder nicht, niemals eine semantiscbe Form und eine daraus resultierende Bestimmtheit, die dem Richter nur noch eine algorichmische Anwendung übrig ließe. Die rechtsfortbildenden Konstruktionsleistungen sind mit den regelanwendenden Interpretationsleistungen, wie die philosophische Hermeneutik zeigt, ${ }^{20}$ immer unauflöslich verwoben. Deshalb stelit sich das Problem der Verfahrensrationalität für die richterliche Entscheidungspraxis und die Rechesdogmatik von neuem und in anderer Weise.

Im Gesetzgebungsverfahren kann eine ins positive Recht eingewanderte Moralität in der Weise zum Zuge kommen, daß die policischen Zielseczungsdiskurse unter den Beschränkungen des Prinzips allgemeiner Zustimmungsfähigkeit, also jenes moralischen Gesichtspunktes stehen, den wir bei der Begründung von Normen beachten müssen. Bei der kontextsensiblen Anwendung von Normen kommt aber die Unparteilichkeit des Urteils nicht schon dadurch zum Zuge, daß wir uns fragen, was alle wollen könnten, sondern: ob wir alle relevanten Aspekte einer gegebeoen Situation angemessen berücksichtigt haben. Bevor wir entscbeiden können, welche Normen, die unter Umständen miteinander kollidieren und dann im Lichte von Prinzipien in eine Rangfolge gebracht werden müssen, auf einen Fall Anwendung finden, muß geklärt sein, ob die Beschreibung der Situation im Hinblick auf alle berührten Interessen angemessen und vollständig ist. Wie Klaus Günther gezeigt hat," bringt sich die praktische Vernunft in Zusammenhängen der Begründung von Normen durch eine Prüfung der Verallgemeinerungsfähigkeit von Interessen, in Zusammenhängen der Anwendung von Normen durch die angemessene und vollscändige Erfassung relevancer Kontexte im Lichtz konkurrierender Regeln zur Geltung. Dem müssen die rechtlichen Verfahren entsprechen, über die die Unparteilichkeit der Rechtsprechung institutionalisiert werden soll.

(3) Mit diesen Überlegunges ziele ich auf die Idee eines gewaltenteilenden Rechtsstaaces, der seine Legitimităt aus einer Unparteilichkeit verbürgenden Racionalität von Geserzgebungs- und Rechtsprechungsverfahren zieht. Damiz ist nicht mehr gewonnen als ein kritischer Maßscab für die Analyse der Verfassungswirklichkeit. Jene Idee tritt freilich einer Realität, die ihr so wenig entspricht, auch nicht bloß abstrakt - in ohnmächrigem Sollen - gegenüber. Vielmehr kennzeichner die ins positive Recht partiell schon eingewanderte Verfahrensrationalität die (nach dem Zusammenbruch des Vernunftrechrs) einzig übriggebliebene Dimension, in der dem gositiven Recht ein Moment Unverfügbarkeit und eine kontingenten Zugriffen entzogene Strukrur gesichert werden kann.

\section{Die Rationalität rechtlich inscitutionalisienter Veffahren: Vorfragen}

(1) Wenn Legitimicät durch Legalität in Gesellschaften unseres Typs möglich sein soll, muß sich der Legalitä́sglauben, dem die kollektiven Gewißheicen von Religion und Metaphysik abhanden gekommen sind, in irgendeinem Sinne auf die "Rationaliäı» des Rechts stützen. Max Webers Annahme aber, daß eine eigenständige,

$20 \mathrm{~J}$. Esser, Vorverständnis und Mechodenwahl in der Rechtsfindung (Frankfure 1972).

2I K. Günther, Das Prinzip der Unparteilichkeit und die funkuonale Bestimutheit des Reches (Ms. 1986). 
moralfreie, dem Recht als solchen innewohnende Rationalitäı der Grund für die legitimierende Kraft der Legalität sei, bat sich nicht bestätigt. Legitimicät verdankt cine in den Formen begründungspflichrigen positiven Rechts ausgeübte Herrschaft stets einem impliziten moralischen Gehalt der formalen Qualitäten des Rechts. Der Formalismus des Rechts darf indessen nicht zu konkretiscisch an bescimmten semantischen Merkmalen festgemacht werden. Legitimierende $\mathrm{Kraft}$ haben vielmehr Verfahren, die Begründungsforderungen und den Weg zu ihrer argumentativen Einlösung institutionalisieren. Die Legitimationsquelle darf zudem nicht einseitig, niche nur am Ore sei es der politischen Gesetzgebung oder der Rechtsprechung gesucht werden. Denn unter Bedingungen sozialstaaticher Politik kann auch der sorgfältigste demokratische Gesetzgeber Justiz und Verwaltung nicht mehr allein durch die semantische Form des Geserzes binden; ohne regulatorisches Recht kommt er nicht aus. Ein im moralisch-praktischen Sinne rationaler Kern rechtlicher Verfahren schält sich erst heraus, wenn man analysiert, wie sich über die Idee der Unparzeilichkeit sowohl der Normbegründung wie der Anrwendung von verbindlichen Regelungen ein konstruktiver Zusammenhang zwischen geltendem Recht, Gesetzgebungsverfahren und Verfahren der Rechtsanweodung herstellt. Diese Idee der Unparteilichkeit bildet den Kern der prakcischen Vernunft. Wenn wir das Problem der unparteilicben Normanwendung hintanstellen, wird die Idee der Unparteilichkeit zunächst unter dem Aspekt der Begründung von Normen in jeoen Moral- und Gerechigkeitstheorien entaltec, die ein Verfahren vorschlagen, wie man praktische Fragen unter dem moralischen Gesichtspunkt beurteílen kann. Die Razionalicät eines solchen reinen, aller Institutionalisierung vorausgehenden Verfahrens bemißr sich daran, ob in ihm der moral point of view angemessen explizier wird.

Zur Zeit sehe ich drei ernsthafte Kandidaten für eine solche prozeduralistische Gerechrigkeitstheorie. Alle gehen aus der kantischen Tradition hervor, aber sie unterscheiden sich nach den Modellen, anhand deren sie das Verfahren unparteilicher Willensbildung erläutern. ${ }^{21}$ John Rawls geht weiterhin vom Modell der vertraglichen Vereinbarung aus und baut in die Beschreibung des Urzustandes diejenigen normativ gehaltvollen Beschränkungen ein, unter denen der rationale Egoismus der freien und gleichen Parteien zur Wahl richtiger Prinzipieo führen muß. Die Fairneß der Ergebnisse wird durch die Prozedur itrres Zustandekommens gewälyrleistet." ${ }^{23}$ Lawrence Kohlberg verwendet statt dessen G.H. Meads Modell der allgemeinen Reziprozitä miteinander verschränkter Perspektiven. An die Stelle eines idealisierten Urzustandes tritt eine ideale Rollenübersahme, die vom moralisch urteilenden Subjekt fordert, sich in die Lage all derjenigen hineinzuversezzen, die von der Inkraftseczung einer fraglichen Norm betroffen sein würden. ${ }^{24}$ Beide Modelle haben meines Erachtens den Nachteil, daß sie dem koguitiven Anspruch moralischer Urteile nicht ganz gerecht werden. Nach dem Modell des Vertragsabschlusses werden moralische Einsichten an rationale Wahjentscheidungen angeglichen, nach dem Modell der Rollenübernahme an empathische Verstehensleistungen. Kari-Orto Apel und ich haben deshalb vorgeschlagen, die moralische Argumencation selbst als das angemessene Verfahren rationaler Willensbildung zu begreifen. Die Prüfung hypochecischer Gelungsansprüche stellt ein solches Verfahren dar, weil sich jeder, der ernsthaft argumentieren will, auf die idealisierenden Unterstellungen einer

\footnotetext{
22 J. Habermas, Gerchugkeıt und Solidanüt, un: W. Edelstein/G. Nunner-Winkler (Hg.), Besummung der Morstirär (Frankfun 1986).

23 J. Rawls, Eine Theoric der Gerecheigkeir (Frankfurt 1975)

24 L. Kohlberg, The Philosophy of Moral Development (San Francisco 1981).
} 
anspruchsvollen Kommunikationsform einlassen muß. Jeder Teilnehmer an einer Argumentationspraxis muß nämlich pragmatisch voraussetzen, daß im Prinzip alle möglicherweise Betroffenen als Freie und Gleiche an einer kooperaciven Wahrheitssuche teilnehmen könnten, bei der einzig der Zwang des besseren Argumentes zum Zuge kommen darf. ${ }^{23}$

Auf die moralcheoretische Diskussion kann ich mich hier nich einlassen; in unserem Zusammenhang genügt die Feststellung, daß es ernsthafte Kandidacen für eine prozeduralistische Gerechsigkeitstheorie gibr. Nur dáann hängr nämlich meine These nicht in der Luft, daß prozeduralisiertes Recht und moralische Begründung von Prinzipien aufeinander verweisen. Legalität kann nur in dern Maße Legitimitär erzeugen, wic die Rechtsordnung reflexiv auf den mir dem Posicivwerden des Rechts entstandenen Begründungsbedarf reagiert, und $z$ war in der Weise, daß juristische Entscheidungsverfahren institutionalisier werden, die für moralische Diskurse durchlässig sind.

(2) Freilich dürfen die Grenzen zwischen Recht und Moral nicht verwischt werden. Die Prozeduren, die Gerechrigkeirstheorien anbieten, um zu erklären, wie man etwas unter dem moralischen Gesichtspunkt beurteilen kann, haben mit rechtlich institutionalisierten Verfahren nur dies gemein, daß die Rationalitär der Verfahren die "Gültigkeit * der verfahrensmäßig erzielten Resultate verbürgen soll. Die juristischen Verfahren nähern sich aber den Forderungen vollständiger Verfahrensrationalität, weil sie mit institutionellen, also unabhängigen Kriterien verknüpft sind, anhand deren sich aus der Perspekcive eines Unbeteiligten feststellen läßt, ob eine Entscheidung regelgerecht zustandegekommen ist oder nicht. Das Verfahren moralischer, rechelich nicht geregelter Diskurse erfüllt diese Bedingung nicht. Hier ist die Verfahrensrationalität unvollständig. Ob erwas unter dem moralischen Gesichtspunkt beurteilt worden ist, läßt sich nur aus der Perspektive von Beteiligten entscheiden. Dazu fehlen externe oder vorgängige Kriterien. Keines dieser Verf́ahren kommt ohne Idealisierungen aus, auch wenn diese - wie die Kommunikacionsvoraussetzungen der Argumentationspraxis - im Sinne einer schwachen transzendentalen Nörigung als unvermeidlich oder alcernativenlos nachgewiesen werden könnten.

Andererseits sind es gerade die Schwächen einer derart imperfekten Verfahrensrationalität, die unter funktionalen Gesichtspunkten verständlich macheø, warum bestimmte Materien rechtlicher Regelung bedürfen und nicht moralischen Regeln des postraditionalen Zuschnitts überlassen bleiben körnen. Wie immer die Prozedur aussieht, nach der wir prüfen wollen, ob eine Norm die zwanglose, d. h. rational motivierte Zustimmung aller möglicherweise Betrofienen finden könnte, sie garanciert weder Unfehlbarkeit noch Eindeutigkeit noch fristgerechtes Zustandekommen des Resultats. Eine autonome Moral verfügr nur über fallibilistische Verfahren der Normbegründung. Dieses hohe $\mathrm{Maß}$ an kognitiver Unbestimmtheit wird zudem dadurch verstärkt, daß mit einer kontextsensiblen Anwendung hochabstrakter Regeln auf komplexe - möglichst angemessen und in ihren relevanten Aspekten möglichst vollständig beschriebene - Situationen eine zusätzliche strukcurelle Ungewißheit verbunden ist. ${ }^{16}$ Dieser kognitiven Schwäche entspricht eine motivationale Schwäche. Jede posttraditionale Moral verlangt eine Distanzierung von den Selbstverständlichkeiten unproblematisch eingewöhnter Lebensformen. Die von der konkreten Sittlichkeit des Alltags entkoppelten moralischen Einsichten führen nicht 
mehr ohne weiteres die motivationale Kraft mit sich, welche die Urteile auch praktisch wirksam werden läßz. Je mehr sich die Moral verinnerliche und auronom wird, umso mehr zieht sie sich in private Bereiche zurück.

In allen Handlungsbercichen, wo Konflikte, bestandswichtige Probleme, gesellschaftliche Materien überhaupt eine eindeutige, fristgerechte und bindende Regelung verlangen, müssen deshalb Rechtsnormen die Unsicherheiten absorbieren, die aufräten, wenn sie einer rein moralischen Verhaltenssteverung überlasscn blieben. Diese Ergänzung der Moral durch zwingendes Recht läßr sich selbst noch moralisch begründen. K. O. Apel spricht in diesem Zusammenhang vom Problem der Zumutbarkeit einer anspruchsvollen universalistischen Moral. ${ }^{27}$ Zumutbar sind nämlich auch moralisch gut begründete Normen nur in dem Maße, wie diejenigen, die ihre Praxis danach einrichcen, erwarten dürfen, daß auch alle anderen sich normenkonform verhalten. Denn nur unter der Bedingung einer allgemein prakrizierten Befolgung von Normen zählen die Gründe, die zu ihrer Rechtfertigung angeführt werden können. Wenn nun von moralischen Einsichten eine praxiswirksame Verbindlichkeit nicht durchgängig enwartet werden kann, ist die Befolgung entsprechender Normen verantwortungsethisch nur zumutbar, wenn sie Rechtsverbindlichkeit erlangen.

Wichcige Merkmale des positiven Rechts werden verständlich, wenn wir das Recht aus diesem Blickrvinkel einer Kompensation der Schwächen autonomer Moral begreifen. Rechtlich institutionalisierte Verhaltenserwartungen erlangen über die Koppelung mit dem staaclichen Sanktionspotential bindende Kraft. Sie erstrecken sich auf das, was Kant den äußeren Aspekt des Handelns nennt, nicht auf Morive und Gesinnungen, die nicht erzwungen werden können. Die professionelle Verwaltung des schriftlich fixierten, öffentlichen und systematisch ausgestalteten Rechts enclaster die privaten Rechrspersonen von dem Aufwand, der bei der moralischen Lösung von Handlungskonfliken dem Einzelnen selbst abverlangt wird. Schließlich verdankt das positive Recht seine konventionellen Züge dem Umstand, daß es durch die Entscheidungen eines politischen Gesetzgebers in Kraft geseczt und im Prinzip beliebig geändert werden kann.

Diese Abhängigkeit von der Politik erklärt auch den instrumentellen Charakter des Rechts. Während Moralnormen stets Selbstzweck sind, sind Rechtsnormen auch Müzel für politische Ziele. Sie dienen nämlich nicht nur wie die Moral der unparteilichen Beilegung von Handlungskonflikten, sondern auch der Umsetzung politischer Programme. Die kollekriven Zielserzungen und die implemencierenden Maßnahmen der Politik verdanken der Rechtsform ihre bindende Kraft. Insofern steht das Recht zwischen Poliak und Moral; und entsprechend verbinden sich, wie Dworkin gezeigt hat, im juristischen Diskurs die Anwendungsargumente der Gesetzesauslegung sowohl mic politischen Ziclsetzungsargumenten wie andererseits mit moralischen Begründungsargumenten.

(3) Die Frage nach der Legitimität der Legalicät hat bisher das Thema Reche und Moral in den Vordergrund gerückt. Wir haben uns klar gemacht, wie das konventionell veräußerlichte Recht und die verinnerlichte Moral einander ergänzen. Mehr als dieses komplementäre Verhältnis interessiert uns jedoch die gleichzeitige Verschränkung von Moral und Recht. Diese kommt dadurch zustande, daß in rechtsstaatichen Ordnungen Mitrel des positiven Rechts in Anspruch genommen werden, um

27 K.O. Apel, Kann der postkancische Suandpunkt der Moralitat noch eınmal in substancielle Sitclichketr aufgehoben werden?, in: W. Kuhimann (Hg.), Moralizăt und Sirlichkeı (Frankfure 1986), $232 \mathrm{H}$. 
Argumentationslasten zu verteilen und Begründungswege zu institutionalisieren, die zu moralischen Argumentacionen hin geöffnet sind. Die Moral schwebt nicht mehr, wie noch die Konstruktion des Vernunftrechts als eines überpositiven Satzes von Normen suggeriert, über dem Recht; sie wandert ins positive Recht ein, ohne darin aufzugehen. Die Moralität, die dem Recht nicht nur gegenübersteht, sondern sich auch im Recht selber festsetzt, ist freilich rein prozeduraler Natur; sie hat sich aller bestimmsen Norminhalte encledigt und zu einem Verfahren der Begründung möglicher Norminhalte sublimiert. So können sich Verfahrensrecht und prozeduralisierte Moral gegenseitıg kontrollieren. In juristischen Diskursen wird die argumencarive Behandlung moralisch-praktischer Fragen auf dem Wege rechticher Instiotionalisierung gleichsam gezähmt; diese wird methodisch durch die Bindung ans geltende Recht, sachlicb im Hinblick auf Themen und Beweislasten, sozial im Hinblick auf Teilnahmevoraussexzungen, Immunitäten und Rollenverteilungen, zeitlich im Hinblick auf Entscheidungsfristen limitier. Umgekehrt wird aber die moralische Argumentation auch als ein offenes Veriahren institutionalisiert, das seiner eigenen Logik gehorcht und so seine eigene Vernünftigkeic kontrolliert. Die rechtliche Verfassung greift nicht ins Innere der Argumentation derart ein, daß diese an den Grenzen des positiven Rechts ins Stocken geriete. Das Recht selbst lizensiert und stimuliert eine Begründungsdynamik, die den Wortlaut des geltenden Rechts auf eine von diesem nicht vorhersehbare Weise auch transzendiert.

Diese Konzeption muß gewiß nach den verscbiedenen Kontexcen rechtswissenschaftlicher, richterlicher, anwaltlicher Diskurse oder auch nach verschiedenen Themenbereichen von moralnahen bis zu rein technischen Fragestellungen differenzien werden. Dann läßt sich auch kritisch die jeweilige Entscheidungspraxis unter dem Gesichtspunkı rekonstruieren, wieweit die rechtlichen Verfahren der Logik der Argumencarion Spielraum geben oder durch implizir mitgeführce externe Beschränkungen das Argumentationsspiel systematisch verzerren. Solche Effekre zeichnen sich natürlich nichı nur an den rechrlichen Veríahrensregelungen selbst ab, sondern auch an der Art, wie diese praktizien werden. Manchrnal bieret sich eine spezielle Klasse von Argumenten für eine solche Rekonstruktion an; in der richterlichen Entscheidungspraxis eigenen sich dafür z.. B. Ureilsbegründungen, die normative Gesichtspunkte zugunsten uncerstellter funktionaler Erfordernisse ausschalten. Allerdings zeige sich gerade an solchen Beispielen, daß die Justiz und das Rechtssystem auf die Gesellschaft gewiß reagieren, ihr gegenüber jedoch nichr autonom sind. $\mathrm{Ob}$ man sich systemischen Imperativen, sei es der Ökonomie oder des Staatsapparates selbst, auch dann beugen muß, wenn sie gut begründere Prinzipien verletzen oder beeinträchtigen, entscheidet sich ja letzelich nicht in den Gerichten, auch nicht in der Rechtsöffentlichkeit, sondern in politischen Kämpfen über den Grenzverlauf zwischen System und Lebenswelt.

Nun haben wir gesehen, daß sich die legitimierende Kraft, die in der Rationalität rechelicher Verfahren ihren Sitz hat, der legalen Herrschaft nicht nur über die Verfahrensnormen der Rechisprechung mitteilt, sondern mehr noch über das demokratische Gesetzgebungsverfahren. Daß der parlamentarische Betrieb einen im moralisch-praktischen Sinne rationalen Kern haben könne, ist freilich auf den ersten Blick nicht so plausibel. Hier scheint es in der Weise um den Erwerb polirischer Macht und um die machtgesteuerte Konkurrenz widerstreitender Interessen zu gehen, daß die parlamentarischen Auseinandersetzungen allenfalls einer empirischen Analyse zugänglich sind, nicht aber einer kritischen Rekonstruktion nach dem Muster der fairen Aushandlung von Kompromissen oder gar der diskursiven Willensbildung. An dieser Stelle kann ich ein befriedigendes Modell nicht anbieten; ich möchte aber auf die lange Reibe prozeßorientierter Verfassungslehren hinwei- 
sen, die einen kritisch-rekonscrukciven Ansazz verfolgen. ${ }^{28}$ Dabei werden die Mehrheitsregel, die parlamentarischen Verfahrensnormen, der Wahlmodus usw. unter dem Gesichtspunkt analysier,, wie weit sie in parlamentarischen Entscheidungsprozessen die gleichmäßige Berücksichtigung aller jeweils berührten Interessen und aller jeweils relevanten Aspekte des Falles sicherstellen können. Eine Schwäche dieser Theorien sehe ich niche im prozeßorienticrten Ansatz als solchem, sondem darin, daß sie ihure normativen Gesichtspunkte nicht aus einer Logik der moralischen Argumentation entwickeln und nicht auf die kommunikativen Bedingungen für eine ungehemmte Begründungsdyoamik anwenden. Im übrigen bildet die innerparlamentarische Willensbildung nur ein schmales Segment des öffentlichen Lebens. Die rationale Qualität der politischen Gesetzgebung hängt nicht nur davon ab, wie gewählte Mehrheiten und geschürzte Minderheiten innerhalb des Parlarnents arbeiten. Sie hängt auch ab vom Niveau der Beteiligung und der Schulbildung, vom Grad der Information und der Schärfe der Arcikulation strittiger Fragen im breiten Publikum. Die Qualität des öffentlichen Lebens wird aligemein bestimmt durch die tassächlichen Chancen, die die politische Öffentlichkeit mit ihren Medien und Institucionen eröffnet.

\section{Schluß}

$\mathrm{Daß}$ die überschießende Idee des Rechesstaats, die ich reformuliert habe, nicht überschwenglich ist, sondern aus dem Boden der Rechtswirklichkeit selbst hervorsprießc, ist schließlich daran zu sehen, daß sich die Autonomie des Rechtssystems allein an dieser Idee bemessen läßr. Weno sich jene Dimension, in der sich die rechtlich institutionalisierten Begründungswege zur moralischen Argumentation hin öffnen, schließen würde, wüßten wir nicht einmal mehr, was Autonomie des Rechis anderes als Systemautonomie bedeuten könnte. Autonomie erwirbt ein Rechtssystem nicht für sich alleine. Autonom ist es nur in dem Maße, wie die für Gesetzgebung und Rechtssprechung institurionalisieren Verfahren unparteiliche Ureils- und Willensbildung garancieren und auf diesem Wege einer ethischen Verfahrensrationalität gleichermaßen in Recht und Politik Eingang verschaffen. Kein autonomes Rechi ohne verwirkliche Demokratie.

28 J. Choper, Judicıal Review and Nacional Poliacal Process (1980); J. H. Ely, Democracy and Diserust (1980). Kritisch dazu: R. D. Parker, The Pest of Consututional Theory - And les Future, Ohio Seate Lar Journal 198x, 223 ff. 